\title{
MicroRNA-27b suppresses Helicobacter pylori-induced gastric tumorigenesis through negatively regulating Frizzled7
}

\author{
YAN GENG ${ }^{1}$, XIAOLAN LU ${ }^{2}$, XIAOKANG WU ${ }^{1}$, LI XUE ${ }^{1}$, \\ XIANGLING WANG ${ }^{1}$ and JIRU XU $\mathrm{XU}^{3}$ \\ Departments of ${ }^{1}$ Laboratory and ${ }^{2}$ Gastroenterology, The Second Affiliated Hospital, Xi'an Jiaotong University, \\ Xi'an, Shaanxi 710004; ${ }^{3}$ Department of Immunology and Pathogenic Biology, Health Science Center, \\ Xi'an Jiaotong University, Xi'an, Shaanxi 710061, P.R. China
}

Received November 3, 2015; Accepted December 11, 2015

DOI: $10.3892 /$ or.2016.4572

\begin{abstract}
MicroRNAs (miRNAs) are novel tools for cancer therapy. Frizzled7 (FZD7) is an important co-receptor in the WNT signaling pathway. The WNT signaling pathway is aberrantly activated in Helicobacter pylori (H. pylori)-infected gastric cancer cells. However, the role of FZD7 in $H$. pylori-induced gastric tumorigenesis remains unknown. In this study, we investigated the potential role of FZD7 in $H$. pylori-induced gastric tumorigenesis and validated the possibility that targeting of FZD7 by specific miRNA inhibits $H$. pylori-induced gastric tumorigenesis. First, we found that FZD7 was significantly induced by $H$. pylori infection in a dose- and time-dependent manner. Knockdown of FZD7 by FZD7 small interfering RNA effectively inhibited $H$. pylori infection-induced cell proliferation of gastric cancer cells. We found that microRNA-27b (miR-27b) was the predicted miRNA for FZD7 and that miR-27b negatively regulated FZD7 expression by targeting the 3 '-untranslated region of FZD7. Furthermore, miR-27b overexpression significantly inhibited $H$. pylori infection-induced cell proliferation and WNT signaling pathway activation in gastric cancer cells. Restoration of FZD7 expression significantly attenuated the inhibitory effect of miR-27b overexpression on cell proliferation and WNT signaling pathway activation. Collectively, our study suggests that FZD7 triggered by $H$. pylori infection contributes to the $H$. pylori infection-induced cell prolifera-
\end{abstract}

Correspondence to: Dr Jiru Xu, Department of Immunology and Pathogenic Biology, Health Science Center, Xi'an Jiaotong University, 76 West Yanta Road, Xi'an, Shaanxi 710061, P.R. China E-mail: jiruxuphd@163.com

Abbreviations: miRNAs, microRNAs; H. pylori, Helicobacter pylori; 3'-UTR, 3'-untranslated region; FZD7, Frizzled7; CagA, cytotoxin-associated gene $\mathrm{A}$

Key words: Helicobacter pylori, gastric cancer, Frizzled7, miR-27b tion that links the WNT. Thus, miR-27b may be a promising molecular target for the treatment of the disease.

\section{Introduction}

Gastric cancer is the fourth most diagnosed cancer and the second leading cause of cancer-related death worldwide (1). A pivotal risk factor for gastric carcinogenesis is Helicobacter pylori (H. pylori) infection (2). H. pylori infection is among the most common infectious diseases. It is considered to be carcinogenic and accounts for approximately $80 \%$ of gastric carcinomas $(3,4) . H$. pylori infection also influences the clinical treatment outcome of gastric cancer (5). However, the association between gastric cancer and $H$. pylori infection and its functional mechanism in gastric cancer remain largely unknown. A better understanding of this molecular mechanism may improve therapeutic prevention and treatment for gastric cancer.

H. pylori is a Gram-negative bacteria that contributes to gastric tumorigenesis mainly through its virulence factor cytotoxin-associated gene A (CagA) (6). The CagA protein has been suggested as an oncogenic protein for gastric cancer $(7,8)$. It has been reported that $H$. pylori infection helps activate multiple oncogenic pathways, such as PI3K/Akt (9-11), WNT/ $\beta$-catenin (12), and Janus kinase signal transducers, as well as activators of the transcription 3 signaling pathway (13). $H$. pylori infection also deactivates tumor suppressors, such as p53 $(14,15)$. Therefore, focusing on oncogenic signaling pathways in $H$. pylori infection-induced gastric carcinogenesis could support novel and promising therapeutic strategies for gastric cancer.

Frizzled7 (FZD7) is a critical receptor for the WNT/ $\beta$-catenin signaling pathway $(16,17)$. Various studies have reported that FZD7 plays an important role in many types of human cancers, including renal cell carcinoma (18), cervical cancer (19), ovarian cancer (17), breast cancer (20), colon cancer (21), and hepatocellular carcinoma (22). It participates in carcinogenesis mainly through promoting the activation of the $\mathrm{WNT} / \beta$-catenin signaling pathway. Aberrant activation of the WNT/ $\beta$-catenin signaling pathway occurs frequently in gastric cancer (23). H. pylori infection also has been implicated 
in this WNT/ $\beta$-catenin signaling pathway (12). These findings suggest that FZD7 may be involved in the WNT/ $\beta$-catenin signaling pathway that is induced by $H$. pylori infection. However, little is known regarding the role of FZD7 in gastric carcinogenesis induced by $H$.pylori infection.

MicroRNAs (miRNAs) are a class of small, noncoding RNAs that negatively regulate gene expression through binding the 3'-untranslated region (UTR) of target gene $(24,25)$. Therefore, miRNAs mediate cancer cell proliferation, differentiation, and metastasis by targeting various genes and regulating numerous signaling pathways (26). Several studies have suggested miRNAs as novel candidates for cancer therapy (27). Although the role of miRNAs in H. pylori-induced gastric carcinogenesis has been widely investigated in recent years, their precise role and target genes remain unclear. In this study, we investigated the role of FZD7 in $H$. pylori-induced gastric carcinogenesis and demonstrated a targeting relationship between FZD7 and miR-27b, a well characterized tumor-suppressive miRNA $(28,29)$. We found that FZD7 was highly upregulated by $H$. pylori infection and was associated with $H$. pylori infection-induced cell proliferation. miR-27b suppressed $H$. pylori infection-induced cell proliferation and the WNT signaling pathway by directly targeting and negatively regulating FZD7 expression. Our study demonstrated that miR-27b/FZD7 is implicated in $H$. pylori-induced gastric carcinogenesis by affecting the WNT signaling pathway.

\section{Materials and methods}

Cell lines. The gastric epithelial-derived cancer cell lines AGS and BGC-823 and human embryonic kidney cell line HEK-293T were obtained from the American Type Culture Collection (ATCC; Manassas, VA, USA). AGS and BGC-823 cells were grown in RPMI-1640 medium (Gibco, Rockville, MD, USA) and HEK-293T cells were cultured in Dulbecco's modified Eagle's medium (DMEM; Gibco). All cultured media were supplemented with $10 \%$ fetal bovine serum (Gibco) and $1 \%$ penicillin-streptomycin mix (Sigma, St. Louis, MO, USA) in a humidified atmosphere containing $5 \% \mathrm{CO}_{2}$ at $37^{\circ} \mathrm{C}$.

H. pylori culture. Standard strain H. pylori 43504, was purchased from the ATCC and cultured on rain-heart infusion plates containing 5\% goat blood and incubated for 3-4 days in a humidified $\mathrm{CO}_{2}$ incubator containing $10 \% \mathrm{CO}_{2}, 5 \% \mathrm{O}_{2}$, and $85 \% \mathrm{~N}_{2}$ at $37^{\circ} \mathrm{C}$. Thereafter, $H$. pylori was collected and re-suspended in RPMI-1640 for a concentration of $3 \times 10^{8}$ colony forming units $/ \mathrm{ml}$. Gastric cancer cells were then infected with H.pylori at a multiplicity of infection (MOI) of 1:25, 1:50, and 1:100 and incubated for 6 and $12 \mathrm{~h}$.

RNA extraction and real-time quantitative PCR analysis. Total RNA from cells was harvested with a miRNeasy kit (Qiagen, Dusseldorf, Germany). For analysis of FZD7 mRNA expression, RNA was reverse transcribed into cDNA using M-MLV Reverse Transcriptase (BioTeke, Beijing, China) and RT-PCR was performed using SYBR Green PCR Master Mix (Applied Biosystems, Carlsbad, CA, USA). For analysis of miR-27b expression, RNA was converted into cDNA using miScript reverse transcription kit (Qiagen), and real-time quantita- tive PCR (RT-qPCR) was conducted using TaqMan miRNA Reverse Transcription kit (Applied Biosystems). GAPDH or U6 small nuclear RNA was used as an internal reference for relative gene expression quantitation. The RT-qPCR reactions were performed in triplicate, and relative gene expression level was determined by using the $2^{-\Delta \Delta C t}$ method.

Western blot analysis. An equal amount of protein from different samples was isolated by sodium dodecyl sulfate-polyacrylamide gel electrophoresis and then transferred onto a PVDF membrane (Bio-Rad, Hercules, CA, USA). The transferred protein on the membrane was confirmed by Ponceau staining solution. Then, the membrane was blocked in $3 \%$ nonfat milk for $1 \mathrm{~h}$ at $37^{\circ} \mathrm{C}$. Primary antibodies, including anti-FZD7 and anti-GAPDH (Santa Cruz Biotechnology, Santa Cruz, CA, USA), were added and incubated at $4^{\circ} \mathrm{C}$ overnight. After three washes with Tris-buffered saline with Tween-20, horseradish peroxidase-conjugated secondary antibodies (1:5,000; Bioss Inc., Beijing, China) were added and incubated for $1 \mathrm{~h}$. The membrane was then developed by use of enhanced chemiluminescence (Pierce, Rockford, IL, USA). Relative protein expression was quantitated by using Image-Pro Plus 6.0 software.

Cell transfection. FZD7 was knocked down by transfection of FZD7 siRNA (Santa Cruz Biotechnology), according to the manufacturer's instruction. Briefly, FZD7 siRNA and transfection reagent were mixed in transfection medium for $45 \mathrm{~min}$. Cells were washed with transfection medium and then incubated with the FZD7 siRNA mixture for $6 \mathrm{~h}$. Normal growth media were added and incubated for $24 \mathrm{~h}$. The old media were then replaced with fresh normal-growth media and further incubated for 24-72 h. Negative control (NC) siRNA was used as a control. For miRNA transfection, miR-27b mimics and NC miRNA (GenePharma, Shanghai, China) were transfected into cells using Lipofectamine 2000 (Invitrogen, Carlsbad, CA, USA) at a concentration of $20 \mathrm{nM}$ and incubated for $48 \mathrm{~h}$. For FZD7 overexpression, pcDNA3.1/ FZD7 vectors were transiently transfected into cells using Lipofectamine 2000 (Invitrogen) for $48 \mathrm{~h}$. The transfection efficiency was finally detected by RT-qPCR or western blot analysis.

Cell proliferation assay. For the MTT assay, cells were seeded into 96-well plates and transfected with FZD7 siRNA, miR-27b mimics, or pcDNA3.1/FZD7 vectors for $48 \mathrm{~h}$, followed by $H$. pylori infection for $12 \mathrm{~h}$. MTT solution $(5 \mathrm{mg} / \mathrm{ml})$ was added at $20 \mu \mathrm{l} /$ well. Dimethyl sulfoxide was added at $200 \mu \mathrm{l} /$ well to dissolve the formazan products. The optical density of the solution was detected using a microplate reader (Bio-Tek Instruments, Winooski, VT, USA) at a wavelength of $490 \mathrm{~nm}$. For the colony formation assay, cells transfected with FZD7 siRNA, miR-27b mimics, or pcDNA3.1/FZD7 vectors followed by $H$. pylori infection were seeded into a 6-well plate in a growth medium containing $0.3 \%$ noble agar (200 cells/ well) to form natural colonies for 14 days. The cells were washed with phosphate buffer saline and fixed with $4 \%$ paraformaldehyde. The plates were stained with crystal violet (Sigma), and the number of colonies was counted and averaged. 
A

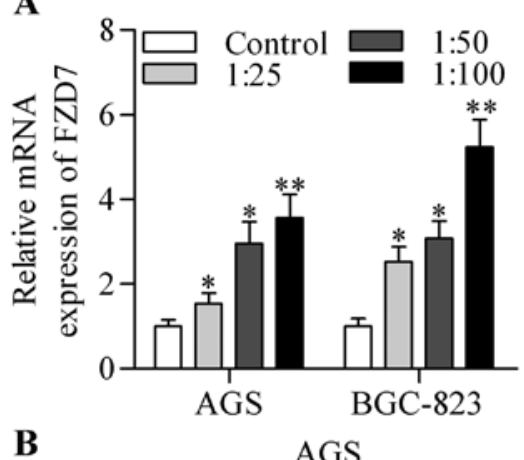

B
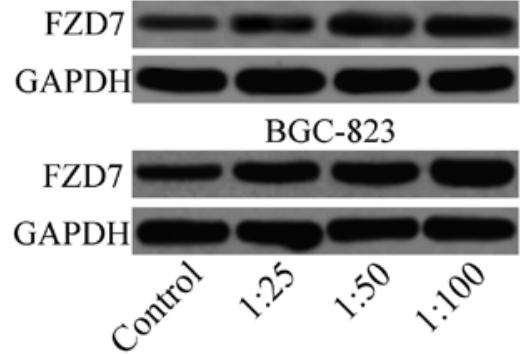

C

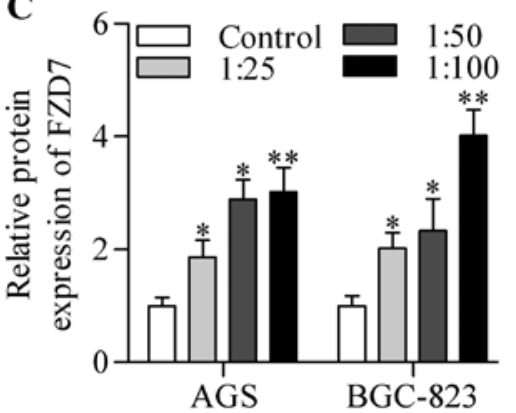

D

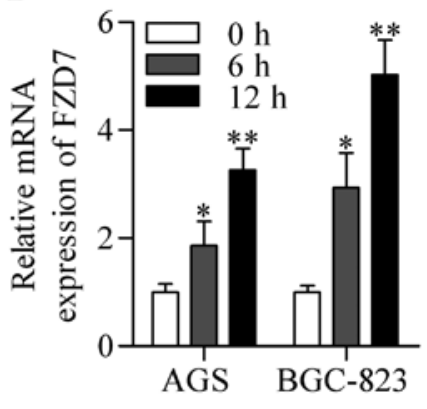

E

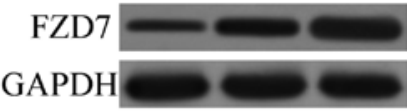

BGC-823

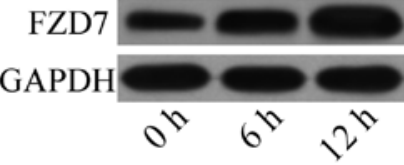

F

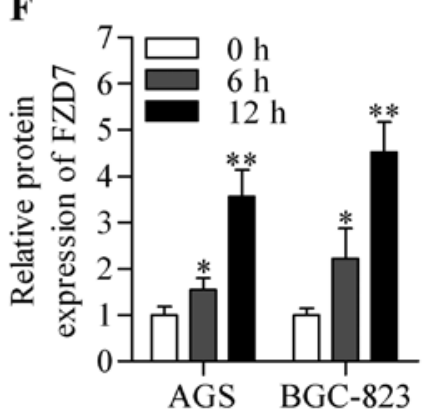

Figure 1. H. pylori infection promotes FZD7 expression. (A) mRNA and (B) protein expression of FZD7 in AGS and BGC-823 cells infected with H. pylori at MOI of 1:25, 1:50, and 1:100, as detected by RT-qPCR and western blot analysis, respectively. Non-infected cells were used as controls. Cells were harvested after infection of $12 \mathrm{~h}$. (C) The relative protein expression of FZD7 in different groups, quantified using Image-Pro Plus 6.0. * $\mathrm{P}<0.05$, ** $\mathrm{P}<0.01$ vs. control. (D) mRNA and (E) protein expression of FZD7 in AGS and BGC-823 cells infected with H. pylori at MOI of 1:100 for 6 or 12 h, as detected by RT-qPCR and western blot analysis, respectively. (F) Quantitative analysis of FZD7 protein expression in different groups. ${ }^{*} \mathrm{P}<0.05$, ${ }^{* *} \mathrm{P}<0.01$ vs. $0 \mathrm{~h}$.

Luciferase reporter assay. The targeted relationship between miR-27b and FZD7 3'-UTR was detected using a dual-luciferase reporter assay. Briefly, the cDNA fragments of FZD7 3'-UTR containing the miR-27b targeted site were inserted into pmirGLO vectors (Promega, Madison, WI, USA). HEK-293T cells were transfected with pmirGLO-FZD7 3'-UTR and miR-27b mimics or NC miRNA and incubated for $48 \mathrm{~h}$. Cells were harvested and lysed. The activity of firefly and Renilla luciferase was tested with a Dual-Luciferase reporter assay kit (Promega). The WNT signaling activity was examined using Tcf luciferase reporter assays. Briefly, gastric cells were co-transfected with a TOPFlash firefly luciferase reporter vector (Addgene, Cambridge, MA, USA) and phRL-TK Renilla luciferase vectors (Promega) together with FZD7 siRNA, miR-27b mimics, or pcDNA3.1/FZD7 vectors, followed by $H$. pylori infection. After $48 \mathrm{~h}$ of transfection, cells were lysed and the activity of firefly and Renilla luciferase was detected, respectively.

Statistical analysis. Quantitative data are shown as mean \pm standard deviation. Statistical analyses were processed with SPSS version 11.5 software (SPSS Inc., Chicago, IL,
USA) with one-way analysis of variance. A p-value of $<0.05$ was regarded as statistically significant.

\section{Results}

H. pylori infection promotes FZD7 expression in gastric cancer cells. To explore whether FZD7 is involved in carcinogenesis related to $H$. pylori infection, we examined its expression level in two gastric cancer cell lines, AGS and BGC-823, infected with $H$. pylori. The results showed that both the mRNA (Fig. 1A) and protein (Fig. 1B and C) expression level of FZD7 were highly upregulated in AGS and BGC-823 cells by $H$. pylori infection in a dose-dependent manner. Further data showed that the mRNA (Fig. 1D) and protein (Fig. 1E and F) expression levels of FZD7 increased in a time-dependent manner. These results indicate that FZD7 was induced by $H$. pylori in gastric cancer cells.

Knockdown of FZD7 inhibits $H$. pylori infection-induced cell proliferation of gastric cancer cells. To investigate the potential biological role of FZD7 in $H$. pylori-induced carcinogenesis, we silenced the expression of FZD7 by using 

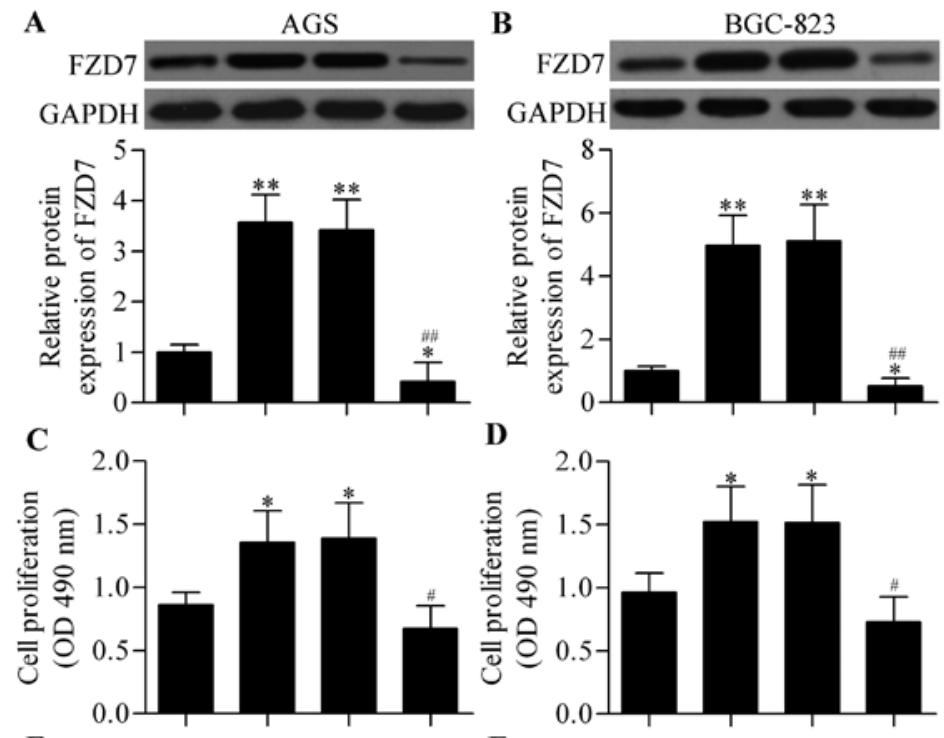

$\mathbf{E}$
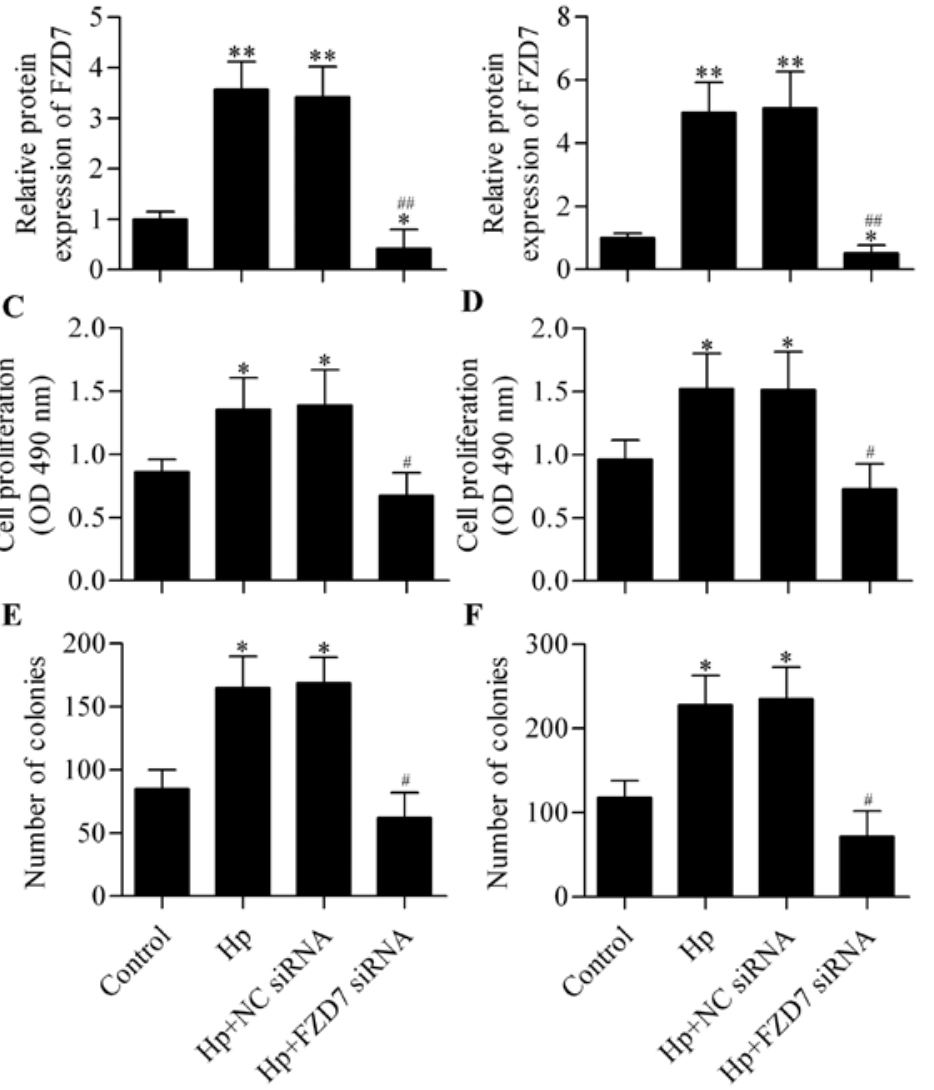

Figure 2. FZD7 is involved in gastric cancer cell proliferation induced by H. pylori infection. Western blot analysis of FZD7 protein expression in (A) AGS and (B) BGC-823 cells. Cells were transfected with FZD7 siRNA or NC siRNA for $48 \mathrm{~h}$ and infected with $H$. pylori at MOI of 1:100 for $12 \mathrm{~h}$. Hp denotes $H$.pylori infection. " $\mathrm{P}<0.05,{ }^{* *} \mathrm{P}<0.01$ vs. control; ${ }^{\# \#} \mathrm{P}<0.01$ vs. Hp+NC siRNA. MTT assay of (C) AGS and (D) BGC-823 cells transfected with FZD7 siRNA or NC siRNA for $48 \mathrm{~h}$ and infected with $H$. pylori at MOI of $1: 100$ for $12 \mathrm{~h}$. ${ }^{*} \mathrm{P}<0.05$ vs. control; ${ }^{\mathrm{H}} \mathrm{P}<0.05 \mathrm{vs}$. Hp+NC siRNA. The effects of FZD7 knockdown on the colony-forming capacity of (E) AGS and (F) BGC-823 cells infected with H. pylori. "P<0.05 vs. control; " $\mathrm{P}<0.05$ vs. Hp+NC siRNA.

A
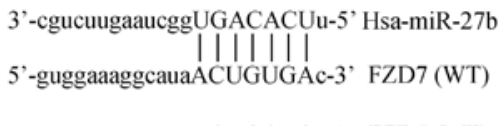

5'-guggaaaggcauaACUGCAGc-3' FZD7 (MT)

B

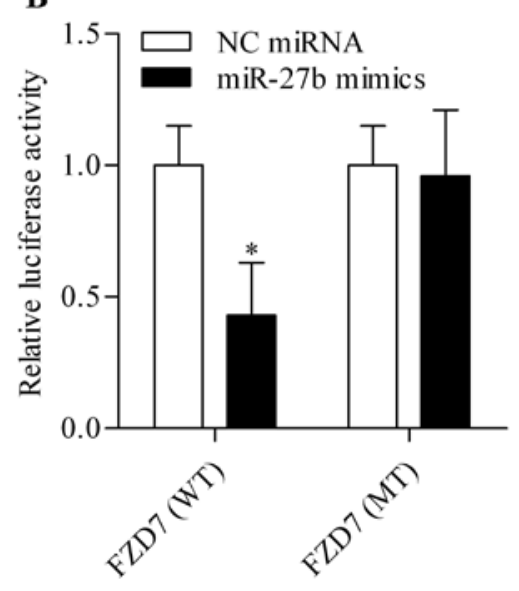

Figure 3. miR-27b directly targets the 3'-UTR of FZD7. (A) Diagram of the predicted miR-27b targeting site within the 3'-UTR of FZD7. (B) Dual-luciferase reporter assay demonstrating the effect of miR-27b on the luciferase activity of the two luciferase reporter vectors containing FZD7 3'-UTR (WT) and FZD7 3'-UTR (MT). "P<0.05 vs. NC miRNA.
FZD7 siRNA (Fig. 2A and B) and detected its effect on gastric cancer cell proliferation with $H$. pylori infection. MTT results showed that $H$. pylori infection significantly promoted gastric cancer cell proliferation and that FZD7 knockdown could reverse this promotion (Fig. 2C and D). Similarly, H. pylori infection markedly upregulated the colony formation of gastric cancer cells, and FZD7 knockdown reversed this upregulation (Fig. 2E and F). These results suggest that the increased expression of FZD7 induced by $H$. pylori infection might contribute to $H$. pylori-related gastric carcinogenesis.

miR-27b directly targets the 3'-UTR of FZD7 and negatively regulates FZD7 expression. miRNAs have emerged as novel tools for cancer treatment because of their regulatory function on gene expression (30). We sought to identify and characterize novel miRNA that could target and regulate FZD7 expression and were thus involved in gastric carcinogenesis related to H. pylori. Through bioinformatic analysis, we found that miR-27b harbored a putative binding site for FZD7 3'-UTR (Fig. 3A). This miRNA attracted our interests because of its critical role in tumorigenesis of various cancer types (29). To validate whether FZD7 is a bona fide target of miR-27b, we performed a dual-luciferase reporter assay using 

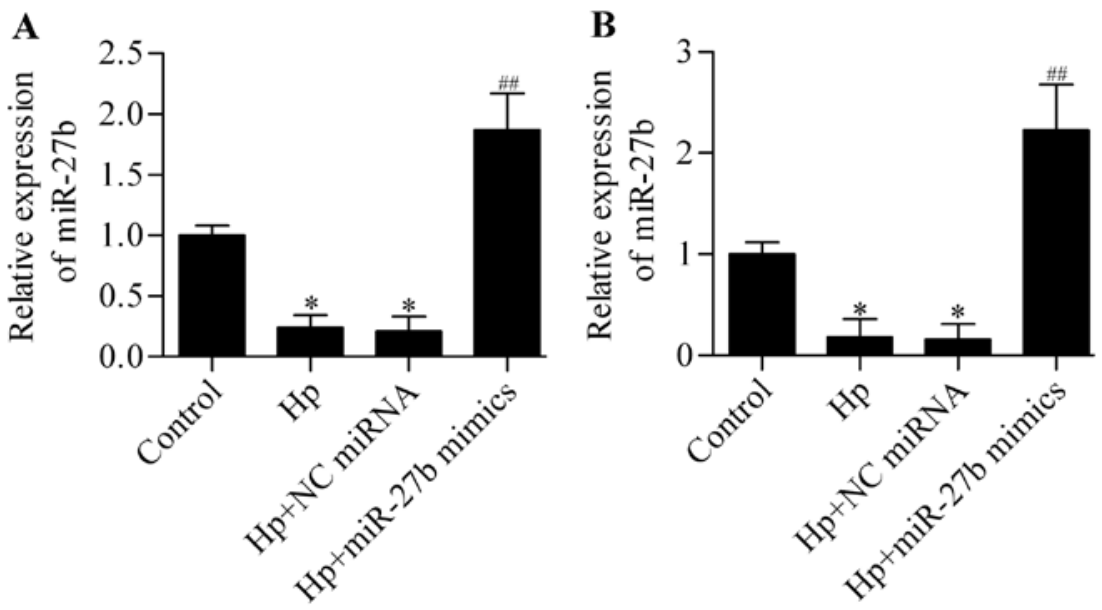

Figure 4. Detection of the miR-27b expression in different groups. RT-qPCR analysis of miR-27b expression in (A) AGS and (B) BGC-823 cells with different treatments. Cells were transfected with miR-27 mimics or NC miRNA for $48 \mathrm{~h}$ and infected with $\mathrm{H}$. pylori for $12 \mathrm{~h}$ at MOI of 1:100. ${ }^{* *} \mathrm{P}<0.01 \mathrm{vs}$. control; ${ }^{\# \#} \mathrm{P}<0.01$ vs. $\mathrm{Hp}+\mathrm{NC}$ miRNA.

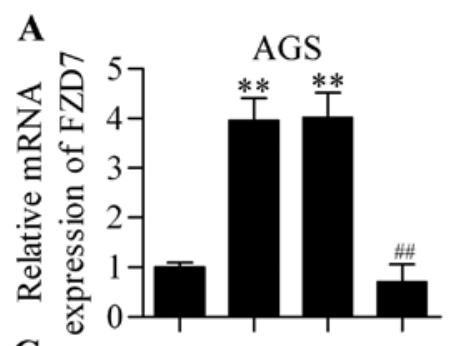

C
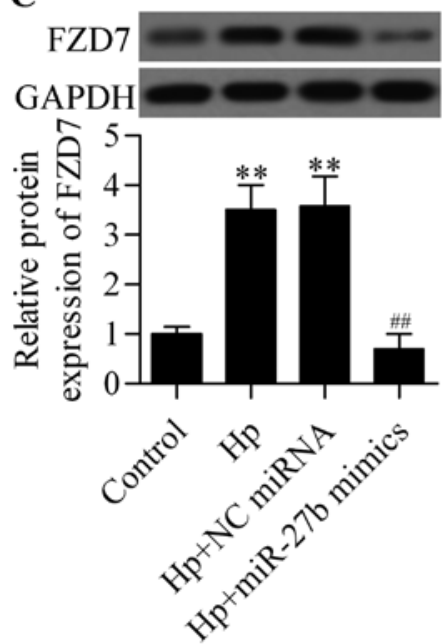

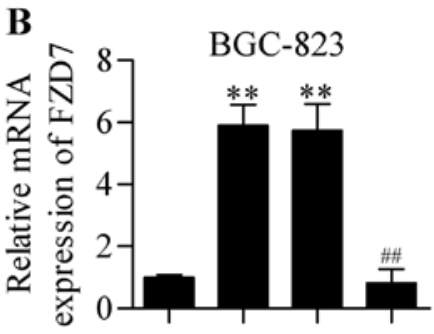

D

FZD7
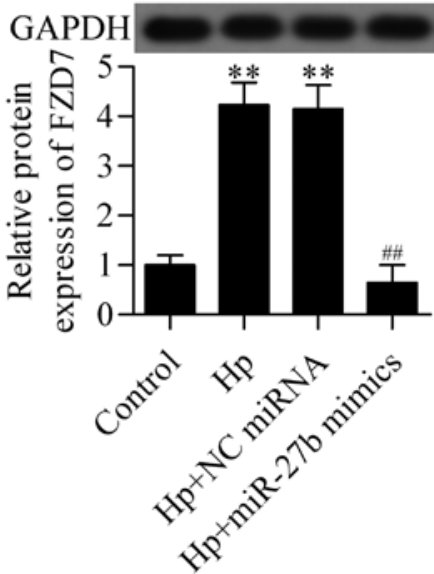

Figure 5. miR-27b regulates the expression of FZD7. RT-qPCR analysis of FZD7 mRNA expression in (A) AGS and (B) BGC-823 cells with different treatments. Western blot analysis of FZD7 mRNA expression in (C) AGS and (D) BGC-823 cells with different treatments. ${ }^{* *} \mathrm{P}<0.01$ vs. control; ${ }^{\# \#} \mathrm{P}<0.01$ versus $\mathrm{Hp}+\mathrm{NC}$ miRNA. Cells were transfected with miR-27 mimics or NC miRNA for $48 \mathrm{~h}$ and infected with $H$. pylori for $12 \mathrm{~h}$ at MOI of 1:100.

two luciferase reporter vectors containing the putative miR-27b binding sites in the wild-type FZD7 3'-UTR (WT) and mutant 3'-UTR (MT). The results showed that co-transfection of HEK-293T cells with miR-27b mimics and luciferase reporter vectors containing the WT FZD7 3'-UTR led to a significant decrease in luciferase activity, compared to NC miRNA transfection (Fig. 3B). In contrast, the luciferase activity of luciferase reporter vectors containing the MT FZD7 3'-UTR was not affected by miR-27b mimics transfection (Fig. 3B). The data imply that miR-27b could target the 3'-UTR of
FZD7 directly. Next, we detected the expression of miR-27b in $H$. pylori-infected cells and found that miR-27b expression was significantly decreased by $H$. pylori infection in AGS and BGC-823 cells, which could be upregulated by transfection of miR-27b mimics (Fig. 4). To investigate whether miR-27b regulated FZD7 expression, we overexpressed miR-27b by transfection of miR-27b mimics in AGS and BGC-823 cells. The results showed that both the mRNA (Fig. 5A and B) and protein (Fig. 5C and D) expression of FZD7 that was upregulated by $H$. pylori infection was significantly decreased by 
A

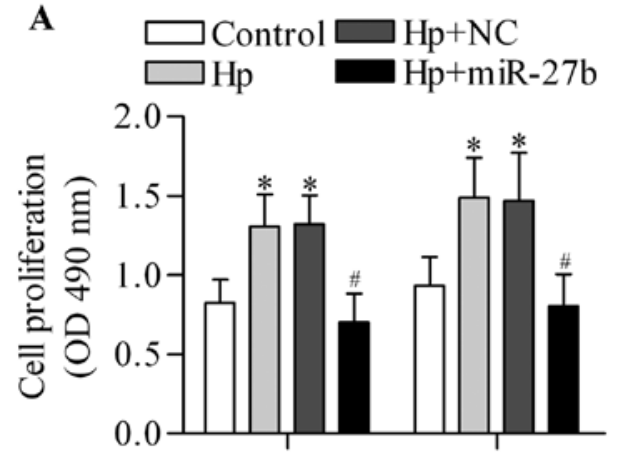

B

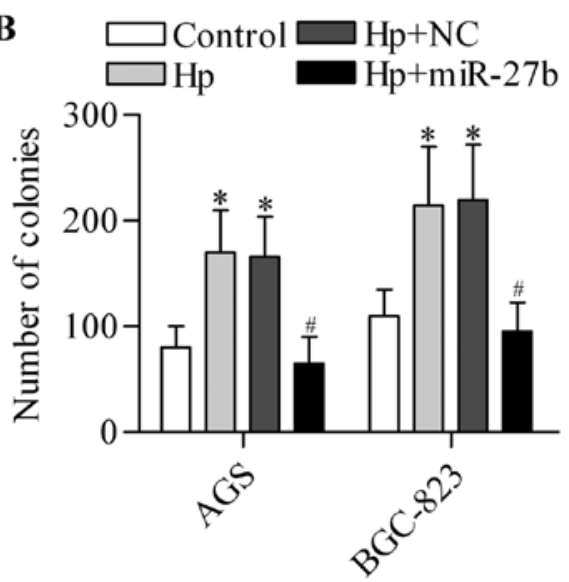

Figure 6. miR-27b is involved in gastric cancer cell proliferation induced by $H$. pylori infection. (A) MTT assay of gastric cancer cells transfected with miR-27b mimics or NC miRNA for $48 \mathrm{~h}$ and infected with $H$. pylori at MOI of 1:100 for $12 \mathrm{~h}$. (B) Effect of miR-27b overexpression on the colony-forming capacity of gastric cancer cells infected with H. pylori. ${ }^{*} \mathrm{P}<0.05$ vs. control; ${ }^{\text {\# }} \mathrm{P}<0.05 \mathrm{vs}$. Hp+NC.

miR-27b overexpression in AGS and BGC-823 cells. Taken together, these results demonstrated that miR-27b regulated FZD7 expression.

The regulation of FZD7 by miR-27b is involved in $H$. pylori-induced cell proliferation. As previously described, $H$. pylori infection significantly promoted the expression of FZD7, and FZD7 was verified as a direct target gene of miR-27b. We thus speculated that miR-27b may play an important role in $H$. pylori-induced cell proliferation by regulating FZD7 expression. To test the hypothesis, we overexpressed miR-27b in $H$. pylori-infected cells and detected its effect on cell proliferation. As expected, the results showed that the cell proliferation (Fig. 6A) and colony formation (Fig. 6B) of gastric cancer cells promoted by $H$. pylori infection could be significantly reversed by miR-27b overexpression. However, restoring expression of FZD7 (Fig. 7A and B) in $H$. pylori-infected cells significantly reversed the inhibitory effect of miR-27b overexpression on $H$. pylori-induced cell proliferation (Fig. 7C and D). In summary, these results indicate that miR-27b inhibited $H$. pylori-induced cell proliferation directly through targeting miR-27b.

Suppression of FZD7 by FZD7 siRNA or miR-27b overexpression inhibits the activation of WNT signaling pathway. A previous study has reported that $H$. pylori infection is associated with activation of the WNT signaling pathway that contributes to carcinogenesis (12). We speculated that the increased expression of FZD7 induced by $H$. pylori infection might contribute to the activated WNT signaling pathway. To test this hypothesis, we suppressed the expression of FZD7 by FZD7 siRNA or miR-27b overexpression and detected their effect on the WNT signaling pathway. The results showed that $H$. pylori infection significantly increased the activity of the WNT signaling pathway, but this promotion was significantly decreased by FZD7 knockdown or miR-27b overexpression in AGS (Fig. 8A) and BGC-823 (Fig. 8B) cells. Further data showed that the inhibitory effect of miR-27b on WNT activity was significantly reversed by restoration of FZD7 expression (Fig. 8C and D). Taken together, the promoted FZD7 expression induced by $H$. pylori infection contributed to the activated WNT signaling pathway, which could be directly inhibited by miR-27b overexpression.

\section{Discussion}

In this study, we demonstrated that $H$. pylori infection significantly upregulated the expression of FZD7 in gastric cancer cells and that this promotion could be markedly reversed by miR-27b overexpression. FZD7 is the critical co-receptor for the WNT signaling pathway. Our result revealed that suppression of FZD7 could block the WNT activation induced by $H$. pylori infection. Therefore, $H$. pylori infection may induce the repression of miR-27b, resulting in overexpression of FZD7 and activation of the WNT signaling pathway, which play important roles in $\mathrm{H}$. pylori-induced gastric tumorigenesis.

$H$. pylori promotes gastric tumorigenesis by facilitating proliferation, angiogenesis, and invasion of cancer cells (31). Various genes and signaling pathways are involved in this process (6). However, the precise molecular mechanism underlying this process remains poorly understood. In this study, we found that FZD7, the critical co-receptor for the WNT signaling pathway, was highly induced by $H$. pylori in AGS and BGC-823 gastric cancer cells. These results indicate that FZD7 may play an important role in $H$. pylori-induced gastric tumorigenesis. Moreover, we found that knockdown of FZD7 significantly decreased the gastric cancer cell proliferation induced by $H$. pylori infection. The results confirmed the pivotal role of FZD7 in $\mathrm{H}$. pylori-induced gastric tumorigenesis. As an important regulator for the WNT signaling pathway, FZD7 has drawn particular interest in tumorigenesis. Studies have revealed that FZD7 is highly expressed in hepatocellular carcinoma and that it contributes to aberrant activation of the WNT signaling pathway $(22,32,33)$. Furthermore, a pharmacological inhibitor of FZD7 showed antitumor effects linked to the inactivated WNT signaling pathway (34). FZD7 is involved in regulating the cell proliferation, invasion, and metastasis of colon cancer cells through the WNT signaling pathway $(21,35)$. Similarly, FZD7 plays critical roles in breast cancer (36) and cervical cancer (19). 

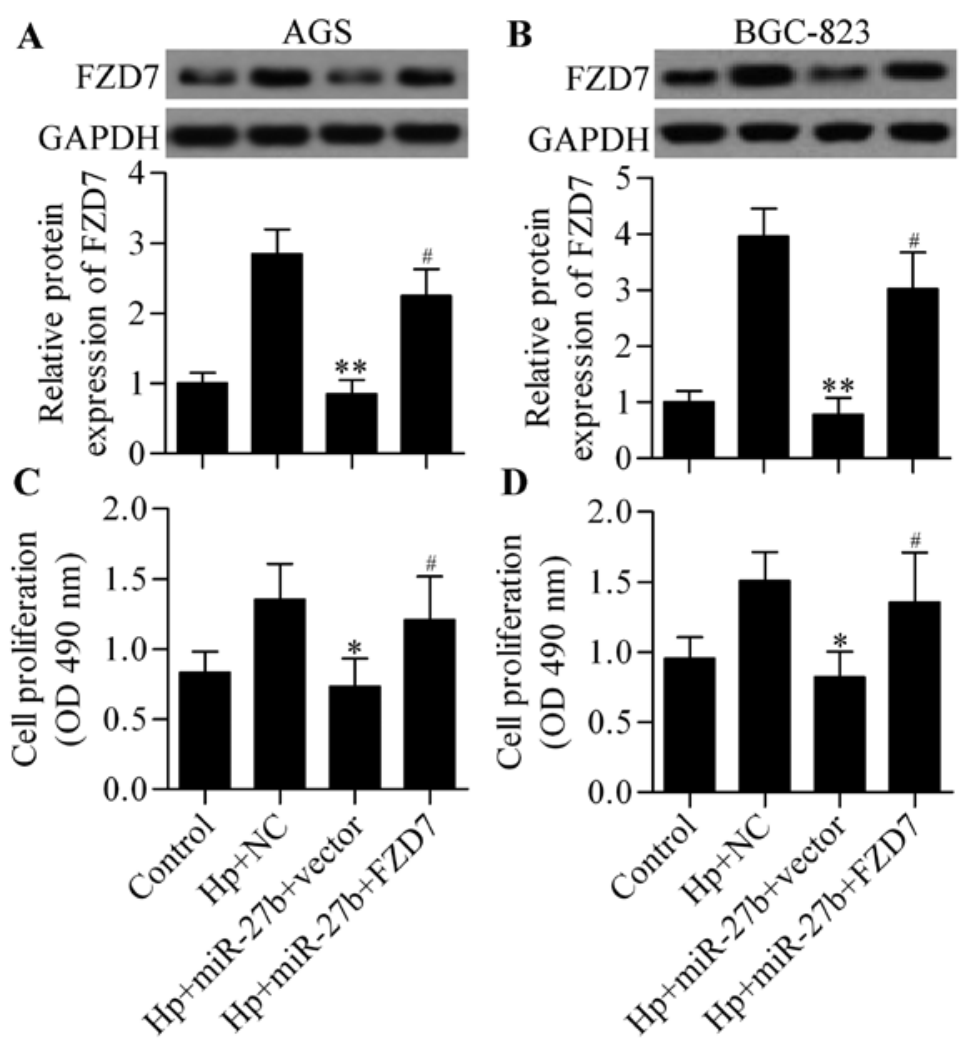

Figure 7. FZD7 overexpression rescues the inhibitory effect of miR-27b on $H$. pylori-induced cell proliferation. Western blot analysis of FZD7 expression in (A) AGS and (B) BGC-823 cells transfected with miR-27b mimics, with or without pcDNA3.1/FZD7. The transfections were incubated for 48 h and infected with $H$. pylori for $12 \mathrm{~h}$ at a multiplicity of infection rate of $1: 100$. Vector, empty vectors. ${ }^{* *} \mathrm{P}<0.01 \mathrm{vs}$. Hp+NC; ${ }^{*} \mathrm{P}<0.05 \mathrm{vs}$. Hp+miR-27b+FZD7. MTT assay of (C) AGS and (D) BGC-823 cells transfected with miR-27b mimics and pcDNA3.1/FZD7 with H. pylori infection. ${ }^{*} \mathrm{P}<0.05$ vs. Hp+NC; ${ }^{*} \mathrm{P}<0.05$ vs. Hp+miR-27b+FZD7.
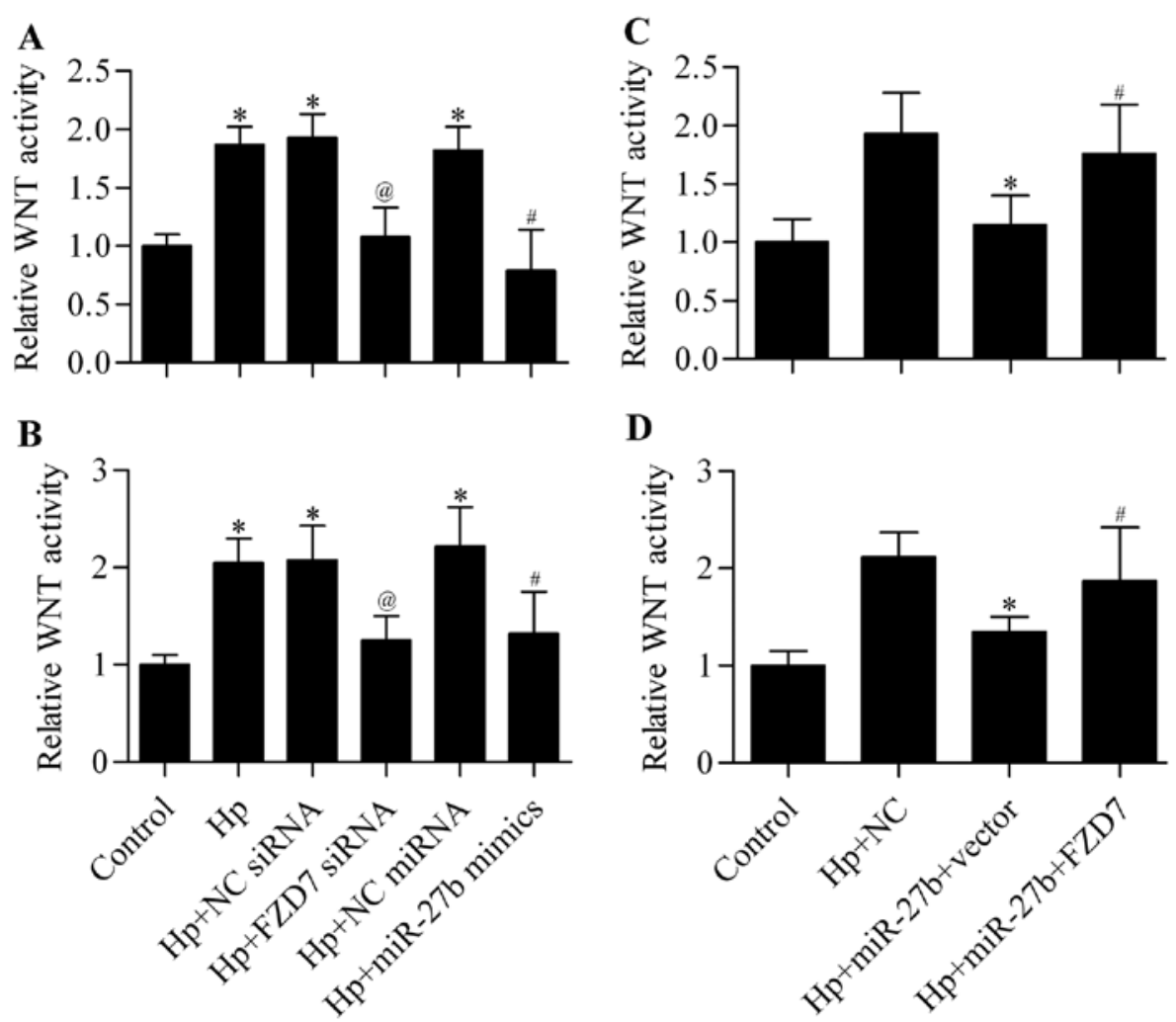

Figure 8. FZD7 regulates WNT activity. Detection of the effect of FZD7 siRNA or miR-27b overexpression on WNT activity in (A) AGS and (B) BGC-823 cells infected with $H$. pylori. ${ }^{*} \mathrm{P}<0.05$ vs. control; ${ }^{\circledR} \mathrm{P}<0.05$ vs. Hp+NC siRNA; ${ }^{\#} \mathrm{P}<0.05$ vs. Hp+NC miRNA. FZD7 overexpression rescues the inhibitory effect of miR-27b overexpression on $H$. pylori-induced cell WNT activation in (C) AGS and (D) BGC-823 cells. ${ }^{*} \mathrm{P}<0.05$ vs. Hp+NC; ${ }^{~} \mathrm{P}<0.05 \mathrm{vs}$. Hp+miR-27b+vector. 
In gastric cancers, it was found that FZD7 was overexpressed and was associated with activation of the WNT signaling pathway (37). Importantly, FZD7-positive gastric cancers are associated with lower survival rates (38). All these findings suggest that FZD7 is dysregulated in cancer development and is positively associated with tumorigenesis. Indeed, FZD7 is considered an emerging and promising molecular target for cancer therapy (39).

Increasing evidence has suggested that miRNAs are emerging tools for cancer therapy because of their negative regulatory effect on target genes (30). Targeting FZD7 by specific miRNA may be a promising and effective therapeutic strategy for cancer. Several studies have tested the hypothesis. For example, miR-23b was found to inhibit the tumorigenic potential of colon cancer cells by regulating pro-metastatic targets, including FZD7 (40). miR-27a suppressed the multiple drug resistance of hepatocellular carcinoma cells by targeting FZD7 and inhibiting the FZD7-mediated WNT signaling pathway (41). Other miRNAs, including miR-199a (42), miR-142-3p (43), and miR-126 (44), were found to be capable of directly targeting and regulating FZD7 expression in cancer cells, implying that these miRNAs are novel and promising approaches for cancer therapy. In this study, we identified miR-27b as a novel miRNA that targets and regulates FZD7 expression. Our results showed that overexpression of miR-27b could inhibit cell proliferation induced by $H$. pylori by suppressing FZD7 expression and by mimicking the effect of FZD7 knockdown via FZD7 siRNA. Additionally, restoring the expression of FZD7 could block the effect of miR-27b overexpression, further confirming that FZD7 was a functional downstream target gene of miR-27b. Recent studies have demonstrated that miR-27b functioned as a tumor suppressor through suppressing the expression of various oncogenic proteins. miR-27b was found to inhibit colorectal cancer progression and angiogenesis by targeting vascular endothelial growth factor C (45). miR-27b suppressed non-small cell lung cancer cell proliferation and invasion by targeting Sp1 transcription factor (29) or LIM kinase 1 (46). Cyclin A2 (47) and ectonucleotide pyrophosphatase/phosphodiesterase family member 1 (48) are also direct target genes of miR-27b in regulating tumorigenesis. Other evidence suggests that miR-27b functions as a tumor suppressor (28,49-51). Most recently, a study demonstrated that miR-27b could target and inhibit cAMP responsive element binding protein 1 which is associated with metastasis and poor outcome in gastric cancer (52). However, this study did not further investigate the function role of miR-27b on gastric cancer. By investigating the expression and functional role of $\mathrm{miR}-27 \mathrm{~b}$ in gastric cancer, our study demonstrated that miR-27b might be a tumor suppressor for $H$. pylori-induced gastric tumorigenesis by modulating FZD7 expression. We found that miR-27b could inhibit gastric cancer cell proliferation by targeting FZD7 and inhibiting the FZD7-mediated WNT signaling pathway involved in $H$. pylori-induced gastric tumorigenesis.

In this study, we also observed that $H$. pylori infection activated the WNT signaling pathway. Other studies have confirm the activation of WNT/ $\beta$-catenin by $H$. pylori (12). However, the underlying mechanism remains obscure. In normal conditions, $\beta$-catenin mainly interacts with E-cadherin at the cell membrane, whereas CagA of $H$. pylori disrupts the complex, resulting in cytoplasmic and nuclear accumulation of $\beta$-catenin for WNT signaling activation (9). Interestingly, in AGS cells lacking the expression of E-cadherin, H. pylori still upregulates the cytoplasmic and nuclear accumulation of $\beta$-catenin, implying that other mechanism are involved in $H$. pylori-induced WNT signaling activation $(9,12)$. Sokolova et al found that $H$. pylori inhibited the activity of GSK-3 $\beta$, leading to increased accumulation of $\beta$-catenin (53). It has also been reported that $H$. pylori infection induced activation of low-density lipoprotein receptor-related protein 6 , another co-receptor of the WNT signaling pathway (54). In this study, we found that $H$. pylori infection induced the expression of FZD7, the co-receptor for WNT signaling pathway. Thus, we speculate that the increased expression of FZD7 may contribute to the activated WNT signaling pathway induced by $H$. pylori infection. Subsequently, we demonstrated that suppression of FZD7 by siRNA or miR-27b significantly blocked the activation of WNT induced by H.pylori infection. Our study thus provides novel insight into the aberrant WNT signaling pathway induced by $H$. pylori infection that involves FZD7.

Our study indicates that $H$. pylori infection triggers the high expression of FZD7 in gastric cancer cells and may contribute to the cell proliferation and WNT activation processes induced by $H$. pylori. Furthermore, we demonstrated that the expression of FZD7 and WNT activation could be inhibited by miR-27b overexpression. We conclude that the miR-27b-FZD7-WNT signaling pathway may be a promising molecular target for the treatment of gastric cancer associated with $H$. pylori infection.

\section{Acknowledgements}

This study was supported by the National Natural Science Foundation of China (no. 81200583 and 81070328).

\section{References}

1. Siegel RL, Miller KD and Jemal A: Cancer statistics, 2015. CA Cancer J Clin 65: 5-29, 2015.

2. Uemura N, Okamoto S, Yamamoto S, Matsumura N, Yamaguchi S, Yamakido M, Taniyama K, Sasaki N and Schlemper RJ: Helicobacter pylori infection and the development of gastric cancer. N Engl J Med 345: 784-789, 2001.

3. Wu WK, Cho CH, Lee CW, Fan D, Wu K, Yu J and Sung JJ: Dysregulation of cellular signaling in gastric cancer. Cancer Lett 295: 144-153, 2010.

4. Shen J, Xiao Z, Wu WK, Wang MH, To KF, Chen Y, Yang W, Li MS, Shin VY, Tong JH, et al: Epigenetic silencing of miR-490-3p reactivates the chromatin remodeler SMARCD1 to promote Helicobacter pylori-induced gastric carcinogenesis. Cancer Res 75: 754-765, 2015.

5. Fukase K, Kato M, Kikuchi S, Inoue K, Uemura N, Okamoto S, Terao S, Amagai K, Hayashi S and Asaka M; Japan Gast Study Group: Effect of eradication of Helicobacter pylori on incidence of metachronous gastric carcinoma after endoscopic resection of early gastric cancer: An open-label, randomised controlled trial. Lancet 372: 392-397, 2008.

6. Yong X, Tang B, Li BS, Xie R, Hu CJ, Luo G, Qin Y, Dong H and Yang SM: Helicobacter pylori virulence factor CagA promotes tumorigenesis of gastric cancer via multiple signaling pathways. Cell Commun Signal 13: 30, 2015.

7. Hayashi T, Senda M, Morohashi H, Higashi H, Horio M, Kashiba Y, Nagase L, Sasaya D, Shimizu T, Venugopalan N, et al: Tertiary structure-function analysis reveals the pathogenic signaling potentiation mechanism of Helicobacter pylori oncogenic effector CagA. Cell Host Microbe 12: 20-33, 2012. 
8. Ohnishi N, Yuasa H, Tanaka S, Sawa H, Miura M, Matsui A, Higashi H, Musashi M, Iwabuchi K, Suzuki M, et al: Transgenic expression of Helicobacter pylori CagA induces gastrointestinal and hematopoietic neoplasms in mouse. Proc Natl Acad Sci USA 105: 1003-1008, 2008.

9. Suzuki M, Mimuro H, Kiga K, Fukumatsu M, Ishijima N, Morikawa H, Nagai S, Koyasu S, Gilman RH, Kersulyte D, et al: Helicobacter pylori CagA phosphorylation-independent function in epithelial proliferation and inflammation. Cell Host Microbe 5: 23-34, 2009.

10. Tabassam FH, Graham DY and Yamaoka Y: Helicobacter pylori activate epidermal growth factor receptor- and phosphatidylinositol 3-OH kinase-dependent Akt and glycogen synthase kinase 3 beta phosphorylation. Cell Microbiol 11: 70-82, 2009

11. Sokolova O, Vieth M, Gnad T, Bozko PM and Naumann M: Helicobacter pylori promotes eukaryotic protein translation by activating phosphatidylinositol 3 kinase/mTOR. Int J Biochem Cell Biol 55: 157-163, 2014

12. Franco AT, Israel DA, Washington MK, Krishna U, Fox JG, Rogers AB, Neish AS, Collier-Hyams L, Perez-Perez GI, Hatakeyama M, et al: Activation of beta-catenin by carcinogenic Helicobacter pylori. Proc Natl Acad Sci USA 102: 10646-10651, 2005.

13. Bronte-Tinkew DM, Terebiznik M, Franco A, Ang M, Ahn D Mimuro H, Sasakawa C, Ropeleski MJ, Peek RM Jr and Jones NL: Helicobacter pylori cytotoxin-associated gene A activates the signal transducer and activator of transcription 3 pathway in vitro and in vivo. Cancer Res 69: 632-639, 2009

14. Wei J, Noto J, Zaika E, Romero-Gallo J, Correa P, El-Rifai W, Peek RM and Zaika A: Pathogenic bacterium Helicobacter pylori alters the expression profile of $\mathrm{p} 53$ protein isoforms and p53 response to cellular stresses. Proc Natl Acad Sci USA 109: E2543-E2550, 2012

15. Wei J, Noto JM, Zaika E, Romero-Gallo J, Piazuelo MB Schneider B, El-Rifai W, Correa P, Peek RM and Zaika AI: Bacterial CagA protein induces degradation of $\mathrm{p} 53$ protein in a p14ARF-dependent manner. Gut 64: 1040-1048, 2015.

16. Abu-Elmagd M, Garcia-Morales $\mathrm{C}$ and Wheeler GN: Frizzled7 mediates canonical Wnt signaling in neural crest induction. Dev Biol 298: 285-298, 2006.

17. Asad M, Wong MK, Tan TZ, Choolani M, Low J, Mori S, Virshup D, Thiery JP and Huang RY: FZD7 drives in vitro aggressiveness in Stem-A subtype of ovarian cancer via regulation of non-canonical Wnt/PCP pathway. Cell Death Dis 5: e1346, 2014

18. Xu R, Zeng S, Xie W, Sun C, Chen YL, Chen MJ and Zhang L: The expression and function of Frizzled-7 in human renal cell carcinoma. Clin Transl Oncol: Aug 5, 2015 (Epub ahead of print) http://dx.doi.org/10.1007/s12094-015-1362-3.

19. Deng B, Zhang S, Miao Y, Zhang Y, Wen F and Guo K: Down-regulation of Frizzled-7 expression inhibits migration invasion, and epithelial-mesenchymal transition of cervical cancer cell lines. Med Oncol 32: 102, 2015.

20. Simmons GE Jr, Pandey S, Nedeljkovic-Kurepa A, Saxena M, Wang A and Pruitt K: Frizzled 7 expression is positively regulated by SIRT1 and $\beta$-catenin in breast cancer cells. PLoS One 9: e98861, 2014.

21. Ueno K, Hazama S, Mitomori S, Nishioka M, Suehiro Y, Hirata $H$ Oka M, Imai K, Dahiya R and Hinoda Y: Down-regulation of frizzled-7 expression decreases survival, invasion and metastatic capabilities of colon cancer cells. Br J Cancer 101: 1374-1381, 2009.

22. Merle P, Kim M, Herrmann M, Gupte A, Lefrançois L, Califano S, Trépo C, Tanaka S, Vitvitski L, de la Monte S, et al: Oncogenic role of the frizzled-7/beta-catenin pathway in hepatocellular carcinoma. J Hepatol 43: 854-862, 2005.

23. White BD, Chien AJ and Dawson DW: Dysregulation of $\mathrm{Wnt} / \beta$-catenin signaling in gastrointestinal cancers. Gastroenterology 142: 219-232, 2012 .

24. Bartel DP: MicroRNAs: Genomics, biogenesis, mechanism, and function. Cell 116: 281-297, 2004.

25. Winter J, Jung S, Keller S, Gregory RI and Diederichs S: Many roads to maturity: microRNA biogenesis pathways and their regulation. Nat Cell Biol 11: 228-234,2009.

26. Dalmay T and Edwards DR: MicroRNAs and the hallmarks of cancer. Oncogene 25: 6170-6175, 2006.

27. Esquela-Kerscher A and Slack FJ: Oncomirs - microRNAs with a role in cancer. Nat Rev Cancer 6: 259-269, 2006.
28. Chiyomaru T, Seki N, Inoguchi S, Ishihara T, Mataki $\mathrm{H}$, Matsushita R, Goto Y, Nishikawa R, Tatarano S, Itesako T, et al: Dual regulation of receptor tyrosine kinase genes EGFR and c-Met by the tumor-suppressive microRNA-23b/27b cluster in bladder cancer. Int J Oncol 46: 487-496, 2015.

29. Jiang J, Lv X, Fan L, Huang G, Zhan Y, Wang M and Lu H MicroRNA-27b suppresses growth and invasion of NSCLC cells by targeting Sp1. Tumour Biol 35: 10019-10023, 2014

30. Jiang C, Chen X, Alattar M, Wei J and Liu H: MicroRNAs in tumorigenesis, metastasis, diagnosis and prognosis of gastric cancer. Cancer Gene Ther 22: 291-301, 2015.

31. Hatakeyama M: Oncogenic mechanisms of the Helicobacter pylori CagA protein. Nat Rev Cancer 4: 688-694, 2004.

32. Merle P, de la Monte S, Kim M, Herrmann M, Tanaka S, Von Dem Bussche A, Kew MC, Trepo C and Wands JR: Functional consequences of frizzled-7 receptor overexpression in human hepatocellular carcinoma. Gastroenterology 127: 1110-1122, 2004

33. Kim M, Lee HC, Tsedensodnom O, Hartley R, Lim YS, Yu E, Merle P and Wands JR: Functional interaction between Wnt 3 and Frizzled-7 leads to activation of the Wnt/beta-catenin signaling pathway in hepatocellular carcinoma cells. J Hepatol 48: 780-791, 2008 .

34. Nambotin SB, Lefrancois L, Sainsily X, Berthillon P, Kim M, Wands JR, Chevallier M, Jalinot P, Scoazec JY, Trepo C, et al: Pharmacological inhibition of Frizzled-7 displays anti-tumor properties in hepatocellular carcinoma. J Hepatol 54: 288-299, 2011.

35. Ueno K, Hiura M, Suehiro Y, Hazama S, Hirata H, Oka M, Imai K, Dahiya R and Hinoda Y: Frizzled-7 as a potential therapeutic target in colorectal cancer. Neoplasia 10: 697-705, 2008.

36. Yang L, Wu X, Wang Y, Zhang K, Wu J, Yuan YC, Deng X, Chen L, Kim CC, Lau S, et al: FZD7 has a critical role in cell proliferation in triple negative breast cancer. Oncogene 30 : 4437-4446, 2011.

37. Kirikoshi H, Sekihara $\mathrm{H}$ and Katoh M: Up-regulation of Frizzled-7 (FZD7) in human gastric cancer. Int J Oncol 19: 111-115, 2001.

38. Schmuck R, Warneke V, Behrens HM, Simon E, Weichert W and Röcken C: Genotypic and phenotypic characterization of side population of gastric cancer cell lines. Am J Pathol 178: 1792-1804, 2011

39. King TD, Zhang W, Suto MJ and Li Y: Frizzled7 as an emerging target for cancer therapy. Cell Signal 24: 846-851, 2012.

40. Zhang H, Hao Y, Yang J, Zhou Y, Li J, Yin S, Sun C, Ma M, Huang Y and Xi JJ: Genome-wide functional screening of miR-23b as a pleiotropic modulator suppressing cancer metastasis. Nat Commun 2: 554, 2011.

41. Chen Z, Ma T, Huang C, Zhang L, Lv X, Xu T, Hu T and Li J: miR-27a modulates the MDR1/P-glycoprotein expression by inhibiting FZD7/ $\beta$-catenin pathway in hepatocellular carcinoma cells. Cell Signal 25: 2693-2701, 2013.

42. Song J, Gao L, Yang G, Tang S, Xie H, Wang Y, Wang J, Zhang Y, Jin J, Gou Y, et al: miR-199a regulates cell proliferation and survival by targeting FZD7. PLoS One 9: e110074, 2014.

43. Deng B, Zhang Y, Zhang S, Wen F, Miao Y and Guo K: MicroRNA-142-3p inhibits cell proliferation and invasion of cervical cancer cells by targeting FZD7. Tumour Biol 36: 8065-8073, 2015.

44. Li Z, Chen P, Su R, Li Y, Hu C, Wang Y, Arnovitz S, He M, Gurbuxani S, Zuo Z, et al: Overexpression and knockout of miR-126 both promote leukemogenesis. Blood 126: 2005-2015, 2015.

45. Ye J, Wu X, Wu D, Wu P, Ni C, Zhang Z, Chen Z, Qiu F, Xu J and Huang J: miRNA-27b targets vascular endothelial growth factor $\mathrm{C}$ to inhibit tumor progression and angiogenesis in colorectal cancer. PLoS One 8: e60687, 2013.

46. Wan L, Zhang L, Fan K and Wang J: miR-27b targets LIMK1 to inhibit growth and invasion of NSCLC cells. Mol Cell Biochem 390: 85-91, 2014.

47. Wang B, Li D, Kovalchuk A, Litvinov D and Kovalchuk O: Ionizing radiation-inducible miR-27b suppresses leukemia proliferation via targeting cyclin A2. Int J Radiat Oncol Biol Phys 90: 53-62, 2014.

48. Takahashi RU, Miyazaki H, Takeshita F, Yamamoto $\mathrm{Y}$, Minoura K, Ono M, Kodaira M, Tamura K, Mori M and Ochiya T: Loss of microRNA-27b contributes to breast cancer stem cell generation by activating ENPP1. Nat Commun 6: 7318, 2015 . 
49. Goto Y, Kojima S, Nishikawa R, Enokida H, Chiyomaru T, Kinoshita T, Nakagawa M, Naya Y, Ichikawa T and Seki N: The microRNA-23b/27b/24-1 cluster is a disease progression marker and tumor suppressor in prostate cancer. Oncotarget 5: 7748-7759, 2014

50. Mu W, Hu C, Zhang H, Qu Z, Cen J, Qiu Z, Li C, Ren H, Li Y, $\mathrm{He} \mathrm{X}$, et al: $\mathrm{miR}-27 \mathrm{~b}$ synergizes with anticancer drugs via $\mathrm{p} 53$ activation and CYP1B1 suppression. Cell Res 25: 477-495, 2015.

51. Veliceasa D, Biyashev D, Qin G, Misener S, Mackie AR, Kishore R and Volpert OV: Therapeutic manipulation of angiogenesis with miR-27b. Vasc Cell 7: 6, 2015.
52. Wang YW, Chen X, Gao JW, Zhang H, Ma RR, Gao ZH and Gao P: High expression of cAMP-responsive element-binding protein 1 (CREB1) is associated with metastasis, tumor stage and poor outcome in gastric cancer. Oncotarget 6: 10646-10657, 2015.

53. Sokolova O, Bozko PM and Naumann M: Helicobacter pylori suppresses glycogen synthase kinase 3beta to promote beta-catenin activity. J Biol Chem 283: 29367-29374, 2008.

54. Gnad T, Feoktistova M, Leverkus M, Lendeckel U and Naumann M: Helicobacter pylori-induced activation of beta-catenin involves low density lipoprotein receptor-related protein 6 and Dishevelled. Mol Cancer 9: 31, 2010. 